\title{
Maturation Process of Photosynthetic Membranes Observed by Proton Magnetic Relaxation and Sorption Isotherm
}

\author{
H. HarańczyK ${ }^{a, *}$, A. Leja ${ }^{a}$, M. JemioŁa-Rzemińska ${ }^{b}$ And K. StrzaŁKA ${ }^{b}$ \\ ${ }^{a}$ Institute of Physics, Jagiellonian University \\ Reymonta 4, 30-059 Cracow, Poland \\ ${ }^{b}$ Faculty of Biochemistry, Biophysics and Biotechnology, Jagiellonian University \\ Gronostajowa 7, 30-387 Cracow, Poland
}

\begin{abstract}
Mild rehydration from the gaseous phase of the developing and mature lyophilized wheat photosynthetic membranes was investigated using hydration kinetics, adsorption isotherm and high power proton relaxometry. Hydration time courses are single exponential for all target air humidities; the hydration time $t^{\mathrm{h}}$ equals to $(11.9 \pm 3.6) \mathrm{h}$ for the mature membranes, and $(17.0 \pm 3.2) \mathrm{h}$ for the developing membranes. The sorption isotherm is sigmoidal in form and well fitted using the Dent model; the mass of water saturating primary binding sites equals $\Delta M / m_{0}=0.033 \pm 0.013$ and $0.025 \pm 0.007$ for the mature and for the developing membranes, respectively, where $m_{0}$ is the dry mass of the sample, and $\Delta M$ is mass of water taken up. Proton free induction decays distinguish: (i) an immobilized proton (Gaussian) component, $S_{0}$, originating from protons of solid matrix of lyophilizate; (ii) a Gaussian component, $S_{1}$, from water bound to the primary water binding sites and localized in proximity of paramagnetic ions; (iii) an exponentially decaying contribution, $L_{1}$, from water tightly bound to lyophilizate surface; and (iv) exponentially decaying loosely bound water pool, $L_{2}$. A significant contribution of water "sealed" in the structure of lyophilized membrane (from the fraction $S_{1}$ and $L_{1}$ ) is detected. The mass of "sealed" water fraction is $\Delta M_{\mathrm{S}} / m_{0}=0.047 \pm 0.023$ and $0.072 \pm 0.021$ for the mature and for the developing membranes, respectively.
\end{abstract}

PACS numbers: 82.56.Na

\section{Introduction}

Drought stress is one of the main factors limiting plant growth. The physiological responses of plants including reduction in photosynthetic activity, stomata closure, alteration in respiratory rates and changes of osmotic potential result, among others, from inhibition of the photosynthetic electron transport and perturbations of membranes structure [1]. Plants differ in their susceptibility to drought stress. Apart from a number of species which are tolerant to a mild water deficit, among higher plants, one can find a plant group resistant to almost complete desiccation named resurrection plants [2]. Not only they can survive equilibration with air of $0 \%$ relative humidity but also they resume normal metabolic processes upon rehydration. A pronounced example of drought and cold resistance are lichens. They can perform photosynthetic activity below $0^{\circ} \mathrm{C}$, at the temperatures below the ice crystallite formation in extracellular spaces. They can take water directly from gaseous phase ([3] and references therein).

While considerable body of evidence has been accumulated about mechanisms involved in protecting plant cells

* corresponding author; e-mail: hubert.haranczyk@uj.edu.pl from dessication-induced damage, less is known about the molecular mechanisms accompanying rehydration of the cells and subcellular structures. A convenient model for studying a recovery from the state of dessication are freeze-dried photosynthetic membranes.

There is still no agreement, how far the lyophilizate of photosynthetic membranes resembles (and/or preserves) the structure of the native membrane. It is still in debate whether photosynthetic membrane lyophilizates retain lamellar phase. However, upon rehydration they return to lamellar phase with the significant contribution of the small membrane fragments from the disrupted lamellae $[4,5]$.

A key factor for understanding the molecular mechanism of recovery of metabolic activity of cell structures during rehydration is knowledge about number and distribution of water binding sites, sequence and kinetics of their saturation, as well as formation of tightly and loosely bound water pools at different steps of rehydration process.

Mild rehydration was performed from gaseous phase in the atmosphere with controlled humidity. The hydration kinetics, the sorption isotherms, and proton free induction decays (FIDs) for different hydration level were analyzed. Photosynthetic membranes were isolated at early stages of greening ( $24 \mathrm{~h}$ of light after 6 days in darkness), 
or from fully developed chloroplasts [6]. For all samples the hydration kinetics was well fitted by the single exponential function, whereas the sorption isotherm revealed the sigmoidal form $[7,8]$, with decreased contribution of high affinity water binding sites as compared to that for lichen thalli $[9,10]$. The hydration isotherm observed by proton FIDs shows the presence of water "sealed" in the structure of lyophilizate, which is not removed by the freeze-drying procedure.

\section{Materials and methods}

Fully developed (mature) thylakoid membranes free of stroma proteins were isolated from wheat (Triticum aestivum L.) var. Jara, grown for seven days in white light $\left(20 \mathrm{~W} \mathrm{~m}^{-2}\right)$. Photosynthetic membranes at earlier stage of greening were isolated from wheat seedlings grown for six days in darkness and subsequently illuminated for $24 \mathrm{~h}$ with white light. The isolation procedure was as described previously [11], however, a $0.067 \mathrm{M}$ phosphate buffer, pH 7.3 containing $0.5 \mathrm{M}$ sucrose was used instead of Tris-HCl buffer. Obtained preparations of photosynthetic membranes were freeze-dried and stored desiccated at $+4^{\circ} \mathrm{C}$ in darkness. Prior to the hydration courses the lyophilizate was incubated for $48 \mathrm{~h}$ over silica gel $\left(p / p_{0}=0 \%\right)$.

The hydration time-courses were performed with controlled humidity, at room temperature $\left(t=22^{\circ} \mathrm{C}\right)$, over the surface of $\mathrm{H}_{3} \mathrm{PO}_{4}\left(p / p_{0}=9 \%\right)$, over the surfaces of saturated solutions of $\mathrm{CaCl}_{2}\left(p / p_{0}=32 \%\right), \mathrm{Na}_{2} \mathrm{Cr}_{2} \mathrm{O}_{7}$ $\left(p / p_{0}=52 \%\right), \mathrm{Na}_{2} \mathrm{~S}_{2} \mathrm{O}_{3}(76 \%), \mathrm{K}_{2} \mathrm{CrO}_{3}(88 \%), \mathrm{Na}_{2} \mathrm{SO}_{4}$ $(93 \%)$, and over a water surface $\left(p / p_{0}=100 \%\right)$. After completing the hydration courses, the dry mass of the photosynthetic membrane was determined upon heating at $70^{\circ} \mathrm{C}$ for $68 \mathrm{~h}$. Higher temperatures were not used as they could cause decomposition of some organic constituents of the membrane [12].

Proton FIDs were recorded on WNS-65 high power relaxometer (Waterloo NMR Spectrometers, St. Agatha, Ontario, Canada). The resonance frequency was $30 \mathrm{MHz}$; the trasmitter power was $400 \mathrm{~W}$; the pulse length $\pi / 2=$ $1.25-1.50 \mu \mathrm{s}$. The high power of the pulse allowed us to observe the total proton signal. FIDs were acquired using Compuscope 2000 card in an IBM clone computer, and averaged over 1000 accumulations. Repetition time was $2 \mathrm{~s}$.

The data obtained were analyzed using the one-dimensional, FID analyzing procedure of the two-dimensional data (in time domain) NMR signal-analyzing program CracSpin written at the Jagiellonian University, Cracow [13].

\section{Results}

The hydration courses for lyophilized wheat photosynthetic membranes performed from the gaseous phase at the relative humidity, $p / p_{0}$, in the range between $9 \%$ and $100 \%$, were fitted well by a single exponential function (see Fig. 1)

$$
\Delta m / m_{0}=B^{\mathrm{h}}+A^{\mathrm{h}}\left[1-\exp \left(-t / t^{\mathrm{h}}\right)\right],
$$

where $\Delta m / m_{0}$ is the relative mass increase, $B^{\mathrm{h}}$ is the saturation hydration level for the very tightly bound water fraction (not removed by incubation at $p / p_{0}=0 \%$ ), $t^{\mathrm{h}}$ is the corresponding hydration time constant, and $A^{\mathrm{h}}$ is the saturation level hydration for bound water. The value of $B^{\mathrm{h}}$, averaged over all hydration courses equals to $0.021 \pm 0.004$ for mature membranes, and $0.022 \pm 0.005$ for developing membranes. Although $B^{\mathrm{h}}$ exceeds that value $(0.009 \pm 0.003)$ for ethylenediamine tetraacetic acid (EDTA) washed photosynthentic membranes [14], it is noticeably lower than that for Antarctic lichens [9, 10]. This may suggest lower density of primary water binding sites on the surfaces of lyophilized photosynthetic membrane compared to the total surface of the cell components of thallus. The hydration time constant for bound water fraction $t^{\mathrm{h}}$, equals $(11.9 \pm 3.6) \mathrm{h}$ and $(17.0 \pm 3.2) \mathrm{h}$ for the mature and for the developing photosynthetic membranes, respectively. A saturation hydration level of bound water fraction, $A^{\mathrm{h}}$, increases gradually with increased humidity.

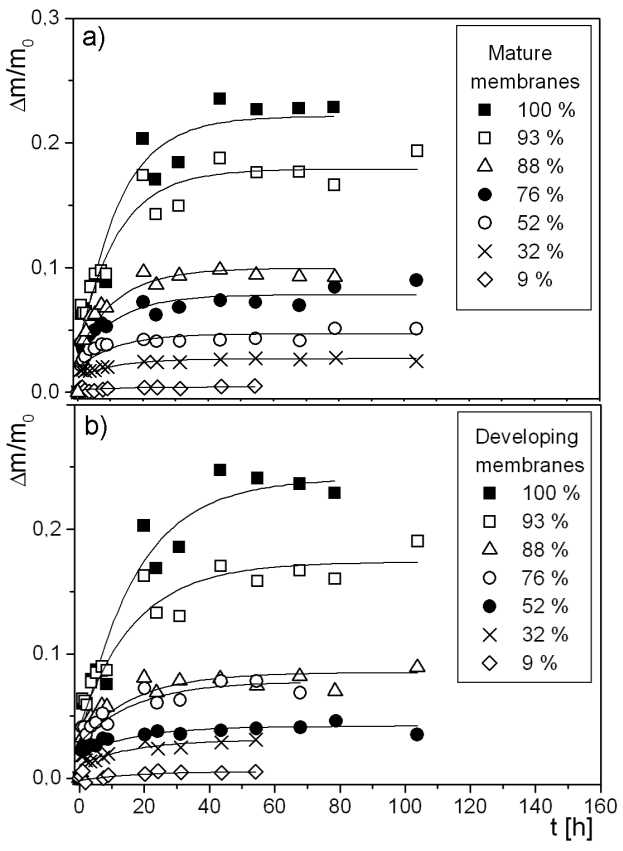

Fig. 1. The rehydration of wheat photosynthetic membrane lyophilizate from gaseous phase at different values of relative humidity, $p / p_{0}$, recorded as relative mass increase, expressed in units of dry mass, $\Delta m / m_{0}$. (a) Mature photosynthetic membranes, (b) developing membranes. The error bars are within the plot symbols.

The total saturation hydration level $C^{\mathrm{h}}=A^{\mathrm{h}}+B^{\mathrm{h}}$, obtained at a given relative humidity, $p / p_{0}$, was taken for construction of sorption isotherm.

For the wheat photosynthetic membrane lyophilizate the adsorption isotherm reveals a sigmoidal form (Fig. 2), which is usually well fitted by the Dent $[7,8]$ and/or the BET [15] model. Both models distinguish two types of 
water binding sites on the surfaces of the system investigated, namely, (i) "primary" water binding sites (directly to the adsorbent surface); and (ii) "secondary", usually weaker, water binding sites (to the primary bound water molecules, to the previous water layers, or to the low affinity surface binding sites). The difference is that the BET model takes a fixed value of the ratio of the number of binding sites covered by $\mathrm{n}$ water molecules to that covered by $n-1$ water molecules, $b=S_{n} /\left.S_{n-1}\right|_{h=1}=1$ (which is an artificial assumption), whereas in the Dent model this ratio may be varied between 0 and 1 (to some extent simulating the droplet formation). The molecular parameters are the escape rate for primary binding sites, $C_{0}$; the escape rate for the secondary binding sites, $C$; and the binding rate, $a$. The parameter $b$ can be expressed as $b=\left(a p_{0}\right) / C$.

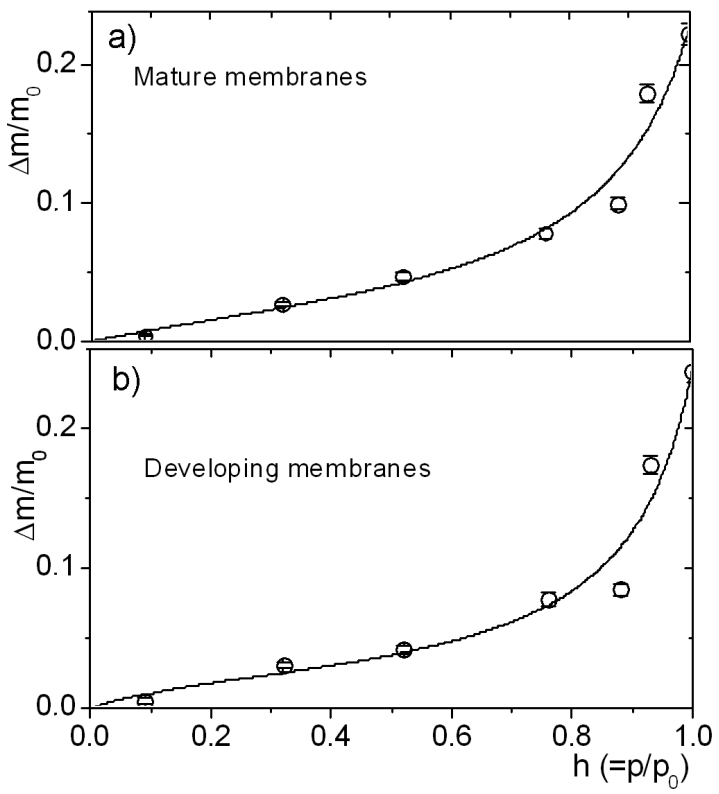

Fig. 2. The sorption isotherms for lyophilizate of wheat photosynthetic membranes. The values of $h\left(=p / p_{0}\right)$ represent the relative humidity and the values of relative mass increase, $\Delta m / m_{0}$, are taken as the saturation values, $C^{\mathrm{h}}=A^{\mathrm{h}}+B^{\mathrm{h}}$, from hydration kinetics Eq. (1). (a) Mature membranes, (b) developing membranes.

The isotherm for both models is described by the formula

$$
\frac{\Delta m}{m_{0}}=\frac{\Delta M}{m_{0}} \frac{b_{1} h}{(1-b h)\left(1+b_{1} h-b h\right)},
$$

where $h$ is relative humidity $p / p_{0}$, expressed in absolute units, and $\Delta M / m_{0}$ is the mass of water saturating primary binding sites. At $h=1$ the contribution of empty primary binding sites, $S_{0}$, is expressed through the reciprocal of $b_{1}$ as $1 / b_{1}=S_{0} /\left.S_{1}\right|_{h=1}=C_{0} /\left(a p_{0}\right)$.

The data fits to Eq. (2) yield the values of sorption isotherm parameters. The mass of water saturating the primary water binding sites, $\Delta M / m_{0}=0.033 \pm 0.013$ for mature membranes and $0.025+0.007$ for developing membranes, which resembles very tightly bound water amount detected in hydration kinetics. For mature membranes the model parameter $b$, indicating the applicability of the Dent model, is equal to $0.863 \pm 0.045$, whereas for developing membranes it is $0.900 \pm 0.027$. The contribution from empty binding sites at $h=1$ is given by $1 / b_{1}=(37.1 \pm 64.3) \%$ (expressed as percentage) for mature membranes and $(16.9+40.6) \%$ for developing membranes. The instability of $1 / b_{1}$ excludes fine discussion on the empty binding site proportion in mature and in developing photosynthetic membranes.

Proton free induction decay of photosynthetic membranes is well fitted by the superposition of two Gaussian components coming from the immobilized protons, with the amplitudes $S_{0}$ and $S_{1}$, respectively; and two exponential components from mobile protons, $L_{1}$ and $L_{2}$,

$$
\begin{aligned}
& \mathrm{FID}(t)=S_{0} \exp \left(-\left(t / T_{G, 0}^{*}\right)^{2}\right)+S_{1} \exp \left(-\left(t / T_{G, 1}^{*}\right)^{2}\right) \\
& \quad+L_{1} \exp \left(-t / T_{2 L_{1}}^{*}\right)+L_{2} \exp \left(-t / T_{2 L_{2}}^{*}\right)
\end{aligned}
$$

where $T_{G, 0}^{*}$ and $T_{G, 1}^{*}$ are the proton spin-spin relaxation times of immobilized components taken as the $1 / \mathrm{e}$-value of Gaussian solid signals; $T_{2 L_{1}}^{*}$ and $T_{2 L_{2}}^{*}$ are the relaxation times of proton liquid fractions, $L_{1}$ and $L_{2}$, respectively. A typical FID is shown in Fig. 3. The 1/e-value instead of the one-half value for solid Gaussian curve allows one a direct comparison between the several characteristic time constants describing FID [16-18].

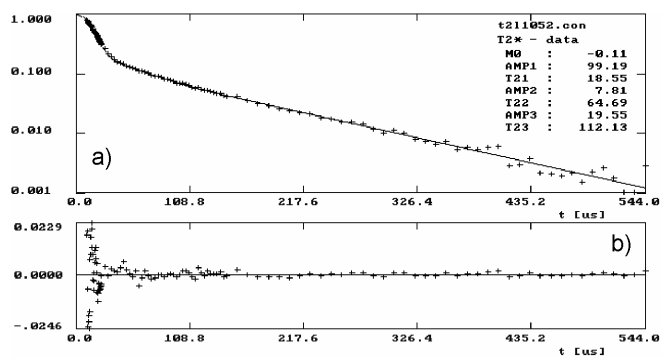

Fig. 3. (a) Proton free induction decay recorded for lyophilizate of mature wheat thylakoid membrane recorded at $30 \mathrm{~Hz}$; the pulse length $\pi / 2=1.25 \mu \mathrm{s}$. The relative mass increase was $\Delta m / m_{0}=0.036$. Solid line represents a least squares fit of Eq. (3). (b) The residual function calculated as a difference between fits and recorded values of FID signal, which for any recorded point does not exceed $2.46 \%$.

The hydration dependences of the proton relaxation times for dehydrated lyophilizates of wheat thylakoids are shown in Fig. 4 . The relaxation time $T_{G, 0}^{*} \approx 20 \mu \mathrm{s}$ (for the shortest Gaussian component) depends only weakly on hydration level of the sample with the value $T_{G, 0}^{*} \approx 20 \mu \mathrm{s}$. This value is close to the value obtained for the other photosynthetic membrane lyophilizates [14], for lichen thalli, and for numerous solid tissues such as dentine, dental enamel, the shells of moluscs, bark and bast (e.g. [17] and references therein). Thus, we assigned the immobilized proton component $S_{0}$ to (i) the solid matrix of the wheat thylakoid lyophilizate. 


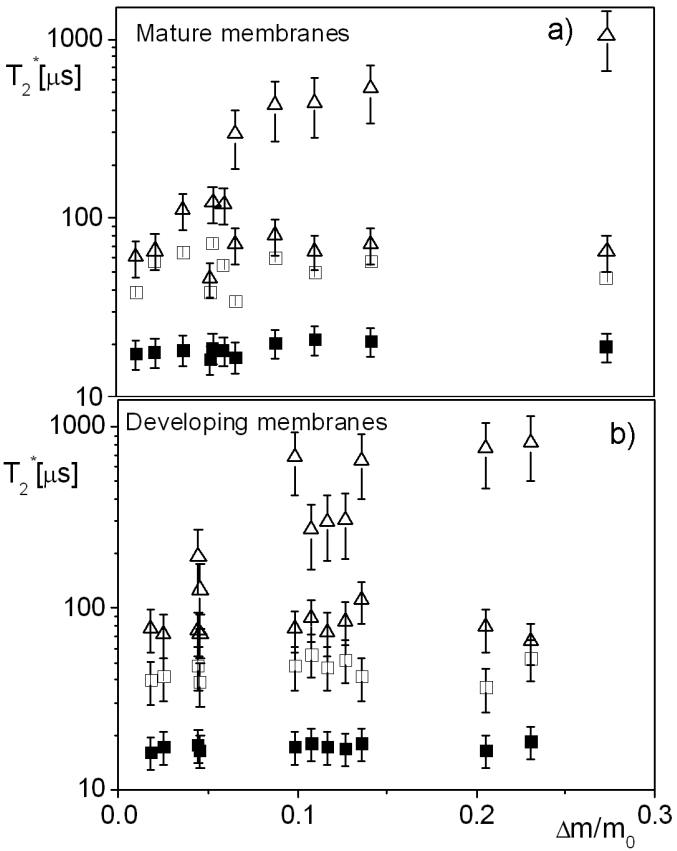

Fig. 4. The hydration dependence of proton FID relaxation times for (a) mature wheat thylakoid membranes, and for (b) developing membranes. Solid Gaussian, $S_{0}$ - closed squares, immobilized water (Gaussian) component, $S_{1}$ - dashed squares, tightly bound water, $L_{1}$ component - dashed triangles, and loosely bound water, $L_{2}$ fraction - open triangles.

At initial stages of rehydration the amplitude of the second (ii) Gaussian component, $S_{1}$, increases with hydration level which means that this component comes from water bound to the surfaces of the lyophilizate. The Gaussian form of the decay and the short value of 1/e-time, $T_{G, 1}^{*}$, suggests that $S_{1}$ component comes from immobilized fraction of water, bound to the primary binding sites on the membrane surface. As the value of $T_{G, 1}^{*}$ does not change monotonically with the hydration level, and equals to $c a .50 \mu \mathrm{s}$, we suggest that it is a homogeneus fraction of water. The contribution of the $S_{1}$ fraction at the hydration level equal to $\Delta m / m_{0}=0$ is called $S_{1,0}$. The Gaussian signal fraction $S_{1}$ vanishes in the case of the thylakoid membranes washed in $1 \mathrm{mM}$ EDTA [14], that also removes the non-functional paramagnetic manganese pool [19-21]. Related to a decrease in partial molar volume and entropy, the ordering effect of water molecules that surround hydrated paramagnetic ion has been already suggested for ions in solution [22]. Evidently, the ordering effect for ion immobilized on the surface should be magnified.

The observed water components are differentiated by their mobility and, thus, for $L_{1}$ and $L_{2}$, by their binding and/or proximity to the thylakoid (immobilized matrix) surfaces.

The amplitude of exponentially decaying mobile fraction $L_{1}$ (iii) increases with the increasing hydration level.
However, its value of spin-spin relaxation time equals $c a$. $80 \mu \mathrm{s}$, and this value does not change monotonically with the hydration level. This resembles the tightly bound water signal for the photosynthetic membrane lyophilizates [14], for lichen thalli, and for solid tissues such as dentine, dental enamel, the shells of moluscs, bark and bast (e.g. [17] and references therein), as well as that of the control porous glass [23]. Thus, we assigned the $L_{1}$ signal component to tightly bound water (presumably first hydration shell). The intercept that yields the contribution of the $L_{1}$ fraction at the hydration level equal to $\Delta m / m_{0}=0$, is called $L_{1,0} / S_{0}$.

The amplitude of the (iv) long exponential component, $L_{2}$, linearly increases with the increasing hydration level with the dependence $L_{2} / S_{0}=(2.879 \pm 0.254) \Delta m / m_{0}$ for the mature membranes, whereas for photosynthetic developing membranes with the dependence: $L_{2} / S_{0}=$ $(2.386 \pm 0.131) \Delta m / m_{0}$ (see Fig. 5). The relaxation time $T_{2 L_{2}}^{*}$ increases with the increasing hydration level (Fig. 4), which suggests that this component may be an average of some proton subsystems being in fast exchange regime [24]. For higher hydration levels the $T_{2 L_{2}}^{*}$ values are of the order of $1 \mathrm{~ms}$. Thus, the $L_{2}$ component is a loosely bound water fraction, or free water. The spinspin relaxation times $T_{2 L_{2}}^{*}$ measured in FID experiment are shortened by $B_{0}$ inhomogeneities [25]:

$$
\frac{1}{T_{2}^{*}}=\frac{1}{T_{2}}+\frac{\gamma \Delta B_{0}}{2}
$$

where $T_{2}$ is spin-spin relaxation time, $\gamma$ is gyromagnetic ratio, and $\Delta B_{0}$ is a change of magnetic field $B_{0}$ within the sample. Although the solid and short exponential components in the FID experiment are not changed by $\Delta B_{0}$ as compared to those measured by the Carr-PurcellMeiboom-Gill (CPMG) echo train, the measured $T_{2 L_{2}}^{*}$ are significantly shortened and may be average $T_{2 L_{2}}^{*}$ values of several loosely bound water pools, e.g. extra- and intramolecular loosely bound water pools.

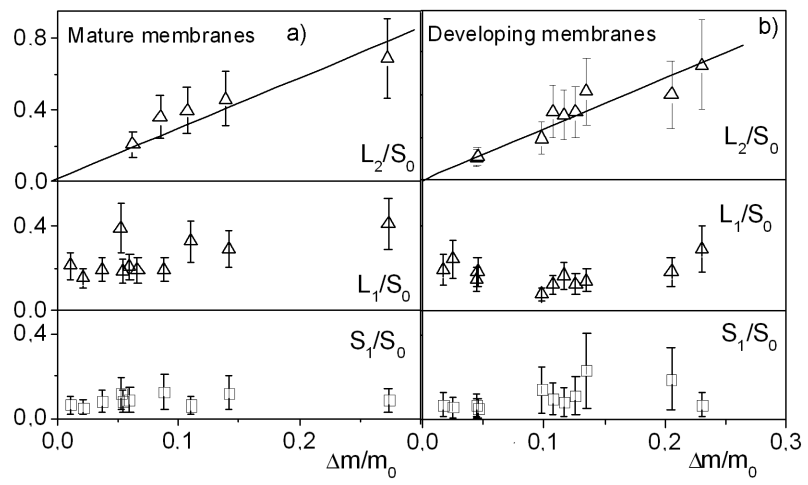

Fig. 5. The $S_{1} / S_{0}, L_{1} / S_{0}$, and $L_{2} / S_{0}$ hydration dependences for (a) mature, and (b) developing wheat thylakoid membranes.

The non-zero contributions of both tightly bound water fractions, $S_{1}$ and $L_{1}$, in dry lyophilizate equal to $S_{1,0}$ and $L_{1,0}$, respectively, and they may suggest the 
presence of "sealed" water pool, which does not leave the wheat photosynthetic membrane during lyophilization procedure [14].

The hydration of total liquid signal, $\left(S_{1}+L_{1}+L_{2}\right) / S_{0}$, expressed in units of solid, $S_{0}$, is well fitted by the linear function (see Fig. 6)

$$
\begin{aligned}
& \left(S_{1}+L_{1}+L_{2}\right) / S_{0}=(3.927 \pm 0.585) \Delta m / m_{0} \\
& \quad+(0.224 \pm 0.064)
\end{aligned}
$$

for mature membranes, whereas for the developing membranes by the function

$$
\begin{aligned}
& \left(S_{1}+L_{1}+L_{2}\right) / S_{0}=(2.988 \pm 0.307) \Delta m / m_{0} \\
& \quad+(0.213 \pm 0.038) .
\end{aligned}
$$

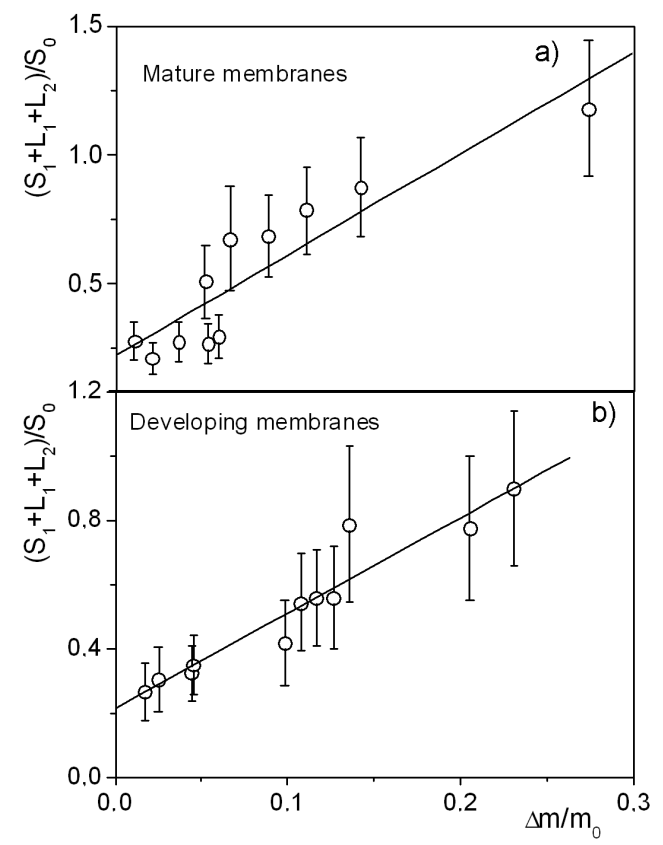

Fig. 6. The $\left(S_{1}+L_{1}+L_{2}\right) / S_{0}$ hydration dependence for (a) mature wheat thylakoid membranes, and for (b) developing membranes rehydrated from lyophilizate. The solid line was calculated from Eq. (5).

The linear form of the total liquid signal hydration dependence suggests the absence of water soluble solid fraction in the samples. Otherwise, the hydration dependence is described by the rational function $[18,26]$.

The slope of the total liquid signal hydration dependence $\left(S_{1}+L_{1}+L_{2}\right) / S_{0}$ yields a scaling factor of NMR signal to mass of water taken up, thus, allows to evaluate the contribution of sealed water fraction in units of dry mass. For the mature membranes the contribution of sealed water $\Delta M_{\mathrm{S}} / m_{0}=0.057 \pm 0.018$ (equal to $\left.S_{1,0}+L_{1,0}\right)$; whereas for the developing membranes the contribution of sealed water equals $\Delta M_{\mathrm{S}} / m_{0}=$ $0.071 \pm 0.015$.

The total liquid signal hydration dependence may be used to construct the NMR-isotherm, with the same sorption parameters $\left(\Delta M / m_{0}, b\right.$, and $\left.b_{1}\right)$ obtained from gravimetry. Only the constant water component, $A$, "sealed" in membrane structures, and the proportionality coefficient $k$, scaling NMR signal in units of water mass, should be fitted. The proportionality coefficient depends on the ratio of the screening effect on the liquid signal to that on the solid signal caused by paramagnetic ions present in the system. This method allows the combination of the classic sorption isotherm with NMR hydration data.

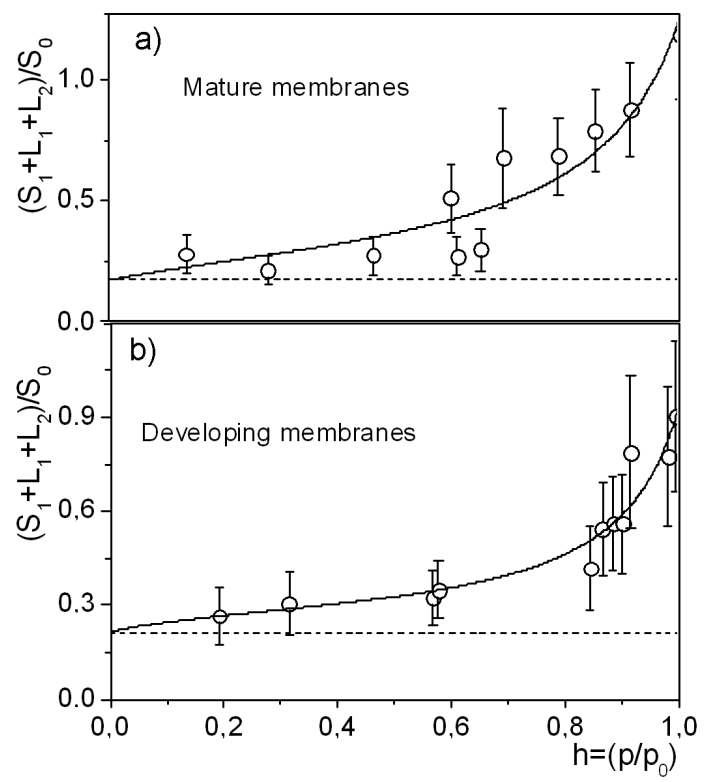

Fig. 7. Sorption isotherm fitted to total FID proton signal for water hydrating (a) mature wheat thylakoid membranes and (b) developing membranes. Open circles - experimental data, solid line - the Dent model Eq. (6) , dotted line — "sealed" water signal.

The normalized amplitude of NMR total liquid signal expressed as a function of $h$ was fitted to the function (see Fig. 7)

$$
\begin{aligned}
& \frac{S_{1}+L_{1}+L_{2}}{S_{0}}(h) \\
& =A+k \frac{\Delta M}{m_{0}} \frac{b_{1} h}{(1-b h)\left(1+b_{1} h-b h\right)} .
\end{aligned}
$$

The solid line was calculated from Eq. (6) with best-fit parameters $k=4.715 \pm 0.601$ and $A=0.174 \pm 0.060$ for mature membranes, and $k=2.968 \pm 0.306$ and $A=0.213 \pm 0.038$ for developing membranes. The values of $A$ and $k$ parameters yield $\Delta M_{\mathrm{S}} / m_{0}=0.037 \pm 0.014$ and $\Delta M_{\mathrm{S}} / m_{0}=0.072 \pm 0.015$ for mature and developing membranes, respectively. These parameter values are consistent with values obtained from the hydration dependence of total liquid signal (Fig. 6, Eq. (5)).

\section{Discussion}

The mass of water saturating the primary water binding sites (very tightly bound water fraction in hydration 
kinetics), $\Delta M / m_{0}$, in lyophilized photosynthetic membranes is lower than in dry lichen thalli [3]. The difference in the number of empty "primary" water binding sites at $h=1$, is not clearly seen because of high errors of the $1 / b_{1}$ parameter fitted. However, both parameters monitoring water binding on membrane surface suggest the decreased "primary" bound water fraction in mature photosynthetic membranes compared to developing ones. This may reflect the proportions of hydrophilic and hydrophobic components in these membranes. Fully developed antenna complexes containing high proportion of photosynthetic pigments may contribute to a more hydrophobic character of the mature photosynthetic membranes.

Proton NMR data revealed the presence of significant fraction of water, which is retained in dry lyophilizate. As both $S_{1}$ fraction and $L_{1}$ fraction contribute to sealed water pool, this pool, equal to $S_{1,0}+L_{1,0}$, is not distinguished by the proximity to the lyophilizate surfaces but it is rather formed in closed spaces present in freeze-dried membranes. Its mass $\Delta M_{\mathrm{S}} / m_{0}$ (in units of $m_{0}$ and averaged over the values obtained using several approaches) equals $\Delta M_{\mathrm{S}} / m_{0}=0.047 \pm 0.023$ for the fully developed membranes, and $\Delta M_{\mathrm{S}} / m_{0}=0.072 \pm 0.021$ for the membranes at the early stage of greening. In the case of both membrane types, the contribution of sealed water, $\Delta M_{\mathrm{S}} / m_{0}$, exceeds twice the contribution of water saturating primary water binding sites, $\Delta M / m_{0}$.

The fraction of "sealed" water may be a water in the lumenal space of thylakoid vesicles, or retained in supramolecular structures formed by proteins and lipids.

The formation of various lipid phase structures can be described by the concept of lipid molecular shape [27]. This approach explains why the most abundant lipid in thylakoid membranes, monogalactosyldiacylglycerol (MGDG), with its small polar headgroup and long polyunsaturated acyl chains, form preferentially $\mathrm{H}_{\mathrm{II}}$ phase, whereas digalactosyldiacylglycerol (DGDG) producing a more cylindrical shaped molecule forms $L_{\alpha}$ phase. The presence of $\mathrm{H}_{\mathrm{II}}$ phase has been detected in the native photosynthetic membranes $[5,28]$ as well as in model systems containing MGDG [29]. Moreover, in mixtures of MGDG and DGDG and in the mixtures of all thylakoid lipids, cubic phase structures are formed $[30,31]$. In this type of structure, the water forms two independent and interwoven branched channels. At each branching point, three branches meet. A monolayer of lipids surrounds each water channel. The hydrocarbon chain from one monolayer meets ones from the other, forming a branched bilayer between water channels. The occurrence of such unique lipid structures in thylakoid membranes seems to offer a good explanation for the high fraction of "sealed" water found in our studies.

In the darkness, etioplasts are present which contain inner membranes differentiated into prothylakoids and one or two prolamellar bodies. In the light, the prolamellar bodies loose their regular structure and disperse into planar membranes. During this process the membrane is first perforated and later the holes close and thylakoids are formed. It was found that in relatively short time, after the $2 \mathrm{~h}$ illumination, the activity of photosystem I (PSI) appeared followed by photosystem II (PSII) activity, which requires about $6 \mathrm{~h}$ light treatment.

Moreover, during development, the amount of lipids per plastid increases three times. The fact that the lag phase for lipids, which is $6-8 \mathrm{~h}$ from the beginning of the light treatment, is longer than that for chlorophyll biosynthesis points to the reorganization into the developing thylakoid.

However, considering the difference between developing and mature membranes, the key factor seems to be their architecture. In the case of matured chloroplasts, there is a profound increase in the number of membranes arranged in the grana stacks [31]. It has been found that mediated by the light harvesting complex ( $\left.\mathrm{LHC}_{\mathrm{II}}\right)$ stacking of thylakoid membranes is observed only in the presence of MGDG, that is considered as a driving force of this process. The recent work of Simidjiev [32] shows that MGDG interacts strongly with $\mathrm{LHC}_{\mathrm{II}}$. Importantly, these are $\mathrm{LHC}_{\mathrm{II}}$ helices, which presumably force MGDG to adopt a bilayer structure. What is still unknown is the mechanism of the membrane stacking in the presence of MGDG. One must bear in mind that uncharged galactose headgroup of MGDG is difficult to hydrate. It could be expected that rather than hydrogen bonding to water, strong hydrogen bonds will be formed between adjacent galactose headgroups. This hydrogen bonding potential may lead to the formation of stacked membranes in the presence of MGDG. Worth mentioning is the fact that thylakoid membrane stacking process is still in progress for developing membranes even when the photosystems activity is already observed. Altogether, such reasoning is consistent with our studies suggesting higher number of empty "primary" water binding sites found for the matured membranes compared to that of developing ones.

\section{References}

[1] H.J. Bohnert, E. Sheveleva, Curr. Opin. Plant Biol. 1, 267 (1998).

[2] D.F. Gaff, Science 174, 1033 (1971).

[3] H. Harańczyk, On Water in Extremely Dry Biological Systems, Wydawnictwo Uniwersytetu Jagiellońskiego, Kraków 2003.

[4] H. Harańczyk, K. Strzałka, T. Bayerl, G. Klose, J.S. Blicharski, Photosynthetica 19, 414 (1985).

[5] H. Harańczyk, K. Strzałka, W. Dietrich, J.S. Blicharski, J. Biol. Phys. 21, 125 (1995).

[6] K. Strzałka, H. Harańczyk, M. Pająk, J.S. Blicharski, Acta Phys. Pol. A 66, 237 (1984).

[7] A.J. Hailwood, S. Horrobin, Trans. Faraday Soc. B 42, 84 (1946).

[8] R.W. Dent, Textile Res. J. 47, 145 (1977).

[9] H. Harańczyk, A. Pietrzyk, A. Leja, M.A. Olech, Acta Phys. Pol. A 109, 411 (2006).

[10] H. Harańczyk, M. Bacior, M.A. Olech, Antarctic Sci. 20, 527 (2008). 
[11] K. Strzałka, G. Majewska, E. Mędrela, Acta Physiol. Plant. 11, 49 (1980).

[12] D.F. Gaff, Oecologia (Berl.) 31, 95 (1977).

[13] W. Wegglarz, H. Harańczyk, J. Phys. D, Appl. Phys. 33, 1909 (2000).

[14] H. Harańczyk, A. Leja, K. Strzałka, Acta Phys. Pol. A 109, 389 (2006).

[15] S. Brunauer, P.H. Emmett, E. Teller, J. Am. Chem. Soc. 60, 309 (1938).

[16] H. Harańczyk, S. Gaździński, M. Olech, New Aspects in Cryptogamic Research, Contribution in Honour of Ludger Kappen. Bibl. Lichenol. 75, 265 (2000a).

[17] H. Harańczyk, S. Gaździński, M.A. Olech, New Phytologist 138, 191 (1998).

[18] H. Harańczyk, W.P. Wȩglarz, S. Sojka, Holzforschung 53, 299 (1999)

[19] T.J. Wydrzynski, S.B. Marks, P.G. Schmidt, Govindjee, H.S. Gutowsky, Biochemistry 17, 2155 (1978).

[20] T.J. Wydrzynski, N. Zumbulyadis, P.G. Schmidt, Govindjee, Biochim. Biophys. Acta 408, 349 (1975).

[21] H.H. Robinson, R.R. Sharp, C.F. Yocum, Biochem. Biophys. Res. Commun. 93, 755 (1980).

[22] H.S. Frank, M.W. Evans, J. Chem. Phys. 13, 507 (1945).
[23] H. Harańczyk, K.G. Soga, R.J. Rumm, M.M. Pintar, Magn. Res. Imag. 9, 723 (1991).

[24] J.R. Zimmerman, W.E. Brittin, J. Phys. Chem. 61, 1328 (1957).

[25] A. Timur, J. Petroleum Technol. 21, 775 (1969).

[26] H. Harańczyk, K. Strzałka, G. Jasiński, K. Mosna-Bojarska, Coll. Surf. A 115, 47 (1996).

[27] J.N. Israelachvili, Intermolecular and Surface Forces, Academic Press, San Diego 1992.

[28] K. Gounaris, A. Sen, A.P.R. Brain, P.J. Quinn, W.P. Williams, Biochim. Biophys. Acta 728, 129 (1983).

[29] D. Latowski, J. Kruk, K. Burda, M. Skrzynecka-Jaskier, A. Kostecka-Gugała, K. Strzałka, Eur. J. Biochem. 269, 4656 (2002).

[30] I. Brentel, E. Selstam, G. Lindblom, Biophim. Biophys. Acta 812, 816 (1985).

[31] E. Selstam, A. Widell Wigge, in: Pigment Protein Complexes in Plastids: Synthesis and Assembly, Eds. L.C. Sundqvist, M. Ryberg, Academic Press, San Diego 1993, p. 241

[32] I. Simidjiev, V. Barzda, L. Mustardy, G. Garab, Biochemistry 37, 4169 (1998). 